\title{
As experiências de quase morte (EOM) podem contribuir para o debate sobre a consciência?
}

\section{Can near death experiences (NDEs) contribute to the debate on consciousness?}

\author{
PETER FenWick ${ }^{1}$ \\ 1 Department Neuroscience, Southampton University, UK. Kings College, Institute of Psychiatry, London University, UK. \\ Tradução: Adriana Sleutjes \\ Revisão da tradução: Alexander Moreira-Almeida
}

Recebido: 10/9/2013 - Aceito: 10/9/2013

\section{Resumo}

A EQM é um estado alterado de consciência que no ocidente inclui uma experiência emocional e de conteúdo estereotipado. Algumas características da experiência são transculturais e sugerem ou um mecanismo cerebral similar ou acesso a uma realidade transcendente. Características individuais da experiência indicam mais persuasivamente para transcendência que para um simples mecanismo cerebral limitado. Além disso, não há, até agora, nenhuma explicação reducionista que possa dar conta satisfatoriamente de algumas dessas características: o encontro com parentes falecidos, a aparente capacidade visual em cegos durante a EQM, a aparente aquisição de dons psíquicos e espirituais após a EQM, relato de cura ocorrida durante uma EQM e experiências verídicas durante a ressuscitação pós-parada cardíaca. Embora uma mente não local pudesse explicar muita das características das EQM, a não localidade ainda não é aceita pela corrente predominante da neurociência. Somente aquelas teorias baseadas num entendimento mais amplo da mente poderiam explicar totalmente a experiência subjetiva dos que vivenciaram uma EQM.

Fenwick P / Rev Psiq Clín. 2013;40(5):203-7

Palavras-chave: Experiência de quase morte, consciência, mente não local, visão mental, dom psíquico.

\begin{abstract}
The NDE is an altered state of consciousness which in the West has stereotyped content and emotional experience. Some features of the experience are transcultural and suggest either a similar brain mechanism or access to a transcendent reality. Individual features of the experience point more persuasively to transcendence than to simple limited brain mechanisms. Moreover there are, so far, no reductionist explanations which can account satisfactorily for some of its features; the meeting of dead relatives, the apparent "sightedness" in the blind during an NDE, the apparent acquisition after an NDE of psychic and spiritual gifts, accounts of healing occurring during an NDE, and of veridical experience during the resuscitation after a cardiac arrest. Although non-local mind would explain many of the NDE features, non locality is not yet accepted by mainstream neuroscience. Only those theories based on a wider understanding of mind could fully explain the subjective experience of the NDEr.
\end{abstract}

Fenwick P / Rev Psiq Clín. 2013;40(5):203-7

Keywords: NDE, consciousness, non-local mind, mind sight, psychic gifts.

\section{Introdução}

Faz mais de 30 anos desde que Raymond Moody publicou sua obra seminal - Life After Life (1975)1. Esse livro descreveu as EQM e trouxe-as à atenção dos públicos leigo e médico. Muitos leigos interpretaram essas experiências como prova de uma extensão da consciência para além da morte (embora o Dr. Moody nunca tenha feito essa afirmação diretamente), enquanto a categoria médica sugeria que mudanças químicas no cérebro eram inteiramente responsáveis pelas experiências.

Noyes e Slymen ${ }^{2}$, na primeira tentativa de uma análise estatística da EQM, encontraram três fatores gerais subjacentes: hiper-vigilância, com aceleração dos pensamentos e visão e audição mais aguçadas; despersonalização, com perda da emoção; e alteração na passagem do tempo, ao mesmo tempo de ter a sensação do self separado ou desprendido do corpo, com sensação de estranhamento ou irrealidade. Em cerca de 1/4 dos sujeitos encontraram também um fator "místico", incluindo sentimento de compreensão ampliada, senso de harmonia e união, sentimento de contentamento e revelação.

Kenneth Ring, psicólogo da Universidade de Connecticut, foi uma das primeiras pessoas a fazer uma análise científica dessas experiências. Ele enumerou as cinco características do que ele chamou de "núcleo" das EQM: sentimento de paz, experiência fora do corpo, entrar na escuridão, visão da luz e entrar na luz. Ele desenvolveu uma escala mais detalhada que incluía o encontro com parentes falecidos, a visão de belas cores ou ouvir música, encontrar um ser ou uma presença e, em alguns casos, uma "revisão da vida". Ele deu pesos diferentes a essas características e, a partir daí, uma EQM poderia ser pontuada - quanto mais alto o escore, mais profunda a experiência ${ }^{3}$.

A sistematização e a padronização entre aqueles que tiveram a experiência se tornaram possível quando Greyson ${ }^{4}$ aplicou o método-padrão de análise fatorial à metade dos seus dados e depois testou os dados ponderados na segunda metade. A partir disso, ele foi capaz de desenvolver a Escala de Greyson, um instrumento de avaliação padrão que pesquisadores agora usam para identificar, para fins de pesquisa, aqueles que tiveram uma EQM. A escala de EQM consiste em quatro grupos de quatro questões, que identificam características cognitivas, afetivas, paranormais e transcendentais da EQM.

\section{Fenomenologia da EOM}

Os fenômenos seguintes são características de EQM nas populações ocidentais:

- calma, ausência de dor;

- saída do corpo (a experiência fora do corpo - EFC);

- viajar por um túnel em direção à luz;

- encontrar "seres" espirituais;

- encontrar parentes falecidos; 
- paisagem bucólica;

- visão retrospectiva da vida;

- reconhecimento de uma barreira ou limite para além do qual não se pode ir;

- volta abrupta ao corpo.

Essas características não ocorrem necessariamente em uma ordem particular e nem todas podem estar presentes numa única experiência. Existem influências culturais também, por exemplo, somente a experiência fora do corpo, "seres" e outros mundos são reportados em todas as culturas. Kellehear ${ }^{5}$ oferece uma excelente revisão sobre esse tema.

\section{Aspectos discrepantes}

Alguns aspectos da EQM - que eu chamo de discrepantes - não se ajustam de modo adequado em uma visão simples, mecanicista e determinista da consciência. Esses aspectos sugerem que a mente deveria ser considerada como tendo efeitos diretos além do cérebro, e assim, tendo um componente não local.

\section{Estados de consciência ampliados na EQM que são supraculturais}

Essa fenomenologia diversificada pode levantar questões sobre as patologias subjacentes à experiência. Atwater ${ }^{6}$ sugeriu que se existisse um mecanismo que produz especificamente EQM, elas seriam semelhantes em diferentes culturas. Porém, há muitas evidências de que o conteúdo de uma experiência mental é em si dependente da cultura na qual ela se dá4. Estados mentais patológicos apresentam ampla variação entre indivíduos e de cultura para cultura. Por exemplo, em estados confusionais tóxicos, tais como as psicoses paranoides em unidade de terapia intensiva, embora possa haver um sentimento paranoide sobrepujante, os detalhes das experiências alucinatórias, delirantes e ilusórias são determinados pelo indivíduo e pela situação em que a experiência ocorre. Assim, a variabilidade é comum e um padrão fixo é menos frequente.

No caso das EQM as similaridades são, assim, importantes e mais significativas que as diferenças. Quando despidos das características culturais, os fenômenos que parecem ser universais nas experiências são os "seres" e a percepção de um outro mundo. A questão importante para pesquisar sobre consciência é se esse mundo, que parece ser acessado tão facilmente de muitas outras maneiras diferentes, é fundamentalmente parte da estrutura de nossas consciências do mesmo modo que é o mundo "real". Uma visão alternativa é que a consciência ampliada desses estados sugere que exista uma dimensão transcendente além do mundo usual à qual os que tiveram uma EQM possuem acesso privilegiado e que pode ser mais do que uma simples questão do funcionamento cerebral. Um livro recente do Dr. Eben Alexander, um neurocirurgião, argumenta que sua EQM foi realmente uma prova do céu ${ }^{7}$.

\section{Falta de um único mecanismo cerebral explicativo}

As condições variadas sob as quais as EQM ocorrem têm levado à discussão de quais circunstâncias são essenciais para tal ser considerada como uma EQM. As EQM surgem de causas orgânicas, por exemplo, lesões ou infecções cerebrais graves, mas fenomenologia similar à da EQM é também encontrada em pacientes que estão doentes, mas não necessariamente próximos à morte. Elas podem também ocorrer em situações em que o sujeito está simplesmente sob intenso medo, mas não doente ou ferido - assim chamada experiência de "medo da morte".

É bastante claro que os mecanismos para a gênese da EQM que são relevantes para os cérebros lesionados não podem explicar as experiências de "medo da morte", de transcendência e outras experiências de EQM, quando não se há evidência de lesão cerebral. Fox ${ }^{8}$ comparou EQM com experiências transcendentes similares e notou que a classificação de uma EQM é "justificada até um certo ponto".
Sartori ${ }^{9}$ enfatiza que as experiências que preenchem os critérios de Moody podem ocorrer também em circunstâncias e em contextos em que não há ameaça ou perigo físico. Ela reforça a sugestão de Fox de que as experiências poderiam ser divididas em experiências de crise e de não crise. Usando essa classificação, Sartori observa que as únicas características de EQM ausentes da experiência de não crise foram a percepção de uma barreira e a visão retrospectiva da vida. É pouquíssimo provável que um único mecanismo poderá explicar todas as experiências.

Experiências transcendentes podem também ocorrer espontaneamente durante a vigília e podem pontuar 7 ou mais na Escala de Greyson, mas sua fenomenologia é mais vaga que dos dois grupos acima, experiências similares que também têm a mesma fenomenologia que as EQM verdadeiras podem ocorrer quando o sujeito está meditando, relaxando ou até mesmo sonhando ${ }^{10}$.

Portanto, fica claro que qualquer teoria que atribua a EQM a um processo orgânico específico deve estar equivocada, a não ser que ela se aplique somente a um subgrupo claramente definido, caso no qual a explicação é somente parcial. Isso é bem revisado por Greyson et al. ${ }^{11}$. As múltiplas situações em que aparece o fenômeno de EQM e a falta de um único e simples sistema explicativo mecanicista com base no cérebro sugere que uma estrutura de consciência mais ampla deve ser considerada.

\section{"Visão mental"}

Adultos cegos desde o nascimento ou que perderam a visão antes dos 5 anos de idade não possuem capacidade de imaginação visual ${ }^{12}$. Seria de se pensar, portanto, que as EQM dessas pessoas seriam bastante diferentes daquelas que possuem a visão.

No entanto, algumas pessoas que perderam um sentido primário, por exemplo, o sentido da visão, têm relatado que parecem ser capazes de, durante uma EQM, usar esse sentido. Em um estudo, $80 \%$ de uma amostra de cegos, incluindo alguns que eram cegos desde o nascimento, relataram experiências visuais durante $\mathrm{EQM}^{13}$. Tem sido proposto que as EQM poderiam ser simplesmente sonhos. Mas cegos congênitos e aqueles que perderam suas visões antes dos 5 anos não têm imagens visuais em seus sonhos. Naqueles que perderam a visão em idades mais avançadas as imagens visuais se esvaem com o tempo.

Dessa forma, a EQM em cegos congênitos parece abrir uma nova trilha sensória que recolhe informações sem um órgão sensorial. Isso é similar a uma visão a distância, em que um sujeito treinado que tem a visão normal é capaz de obter informações visuais acerca de um alvo distante ${ }^{14}$. Parece improvável que existam dois mecanismos diferentes para esses fenômenos semelhantes. De qualquer forma, não tenho conhecimento de nenhum estudo realizado sobre visão a distância em cegos, então não é possível saber se o mecanismo é de fato o mesmo. A relevância para a consciência é mais uma vez clara na medida em que ela sugere que a mente deve estender-se não localmente e obter informações além do alcance dos sentidos.

\section{Transformação após uma EOM}

Qualquer acontecimento que ameace a vida pode, certamente, produzir mudança na personalidade, mas os dados indicam que aqueles que passaram pelas EQM são afetados mais positivamente pelas mudanças que aqueles que passaram por experiências similares de ameaça à vida, mas não tiveram EQM. Greyson ${ }^{15}$ relatou que, em 272 pacientes que tiveram um "encontro com a morte", 61 deles (22\%) tiveram EQM. Observou-se que eles estavam menos perturbados psicologicamente após a experiência que aqueles que não tiveram EQM.

Dois estudos de pacientes que tiveram parada cardíaca são suficientemente abrangentes para sustentar isso. Van Lommel et al. ${ }^{16}$ mediram a qualidade de vida dos sujeitos pós-evento, usando o Life Change Questionnaire. As mudanças nas atitudes sociais e religiosas foram afetadas positivamente. $\mathrm{O}$ medo da morte foi reduzido, e a busca por significado pessoal, o interesse em autocompreensão e a apreciação das coisas comuns foram aumentados. Um estudo menor 
de Schwaninger ${ }^{17}$, usando o mesmo questionário, encontrou essencialmente os mesmos resultados. (Para uma revisão completa, ver Noyes et al. ${ }^{18}$.) Acredita-se que um número de fatores antes e durante a experiência influencia o desenvolvimento, a importância e o alcance dos efeitos posteriores, incluindo as circunstâncias, proximidade da morte e, particularmente, a profundidade da experiência ${ }^{16,17,19}$. Existe também uma relação entre elementos específicos de EQM e seus efeitos posteriores: experiência fora do corpo, por exemplo, sugere aos que a experienciaram a possibilidade de continuação da consciência após a morte e é um modelo para a alma que deixa o corpo $^{20}$. Traços de personalidade podem também ser importantes. Estudos têm examinado tanto as características psicológicas normais quanto as patológicas. Basicamente, a saúde mental de sujeitos que tiveram EQM não se distingue da dos grupos de comparação. Existem algumas opiniões de que a capacidade para absorção psicológica ou tendência à fantasia aumenta a chance de ter uma EQM, mas as evidências não são conclusivas ${ }^{21}$. (Para uma revisão ver Holden ${ }^{22}$.) No entanto, existe evidência que qualquer experiência transcendente profunda pode produzir mudanças espirituais positivas, mesmo se ela não se ajustar claramente à fenomenologia da EQM ${ }^{7}$.

Pessoas que tiveram EQM frequentemente relatam aumento subsequente de experiências paranormais, como pré-cognição, intuição, clarividência, telepatia, experiência fora do corpo, habilidade de cura, perceber aura ou contatar espíritos. No entanto, nenhum estudo de meu conhecimento testou objetivamente as alegações de paranormalidade, assim a validade delas permanece subjetiva. Se fosse de fato demonstrada sua validade, uma mente com propriedades não locais deve ser postulada para explicá-las.

Alguns também descrevem mudanças nos estados de consciência e, em nossa amostra, a ocorrência de fenômenos semelhantes à kundalini, que usualmente tem a forma de choques ou solavancos que se irradiam pelo corpo, e o conhecimento da pessoa de que ela estava em um caminho progressivo que parecia levar a uma expansão da consciência ${ }^{10,18}$.

Há também alegações de cura por meio da própria experiência. O caso mais notório e controverso desse tipo é o descrito por Mellon Thomas Benedict, que alega que ele estava morto por 1 hora durante a qual ele teve uma EQM, e após isso ele afirma que foi curado de um câncer (http://www.mellen-thomas.com/quotes.html). Em um estudo prospectivo de boa qualidade de pacientes em unidade de terapia intensiva e de parada cardíaca em unidades coronarianas, Sartori ${ }^{9}$ observou que $13 \%$ dos pacientes que tiveram EQM relataram cura espontânea após suas EQM, enquanto nenhum paciente controle o fez. Um desses pacientes tinha tido uma contratura na mão por 60 anos, após uma bem documentada paralisia cerebral leve e uma hemiparesia espástica de sua mão direita. Ele teve uma EFC durante sua EQM, na qual ele era capaz de mover a mão dele. Durante a recuperação, ele conseguia de fato mover normalmente sua mão anteriormente contraída, algo que não poderia ter ocorrido sem uma cirurgia especial para liberar a contratura. A cura espontânea está bem documentada na literatura ${ }^{23}$, mas geralmente é vista como intervenções do sistema imune, resultando, por exemplo, na regressão de cânceres. Claramente, esse mecanismo não pode explicar todos os casos e a EQM poderia ser vista como um bom modelo para investigar os efeitos mais amplos dessas experiências. $\mathrm{Na}$ literatura esotérica e sobre meditação existem referências frequentes ao desenvolvimento de poderes especiais (siddhis) após treinamento espiritual ou experiência espiritual profunda. Seria razoável aceitar que a EQM é uma experiência desse tipo e pode também resultar em mudanças nas experiências subjetivas.

\section{Relatos de experiências verídicas}

Percepções verídicas são bem revisadas por Holden ${ }^{22}$. Ela define isso como qualquer percepção visual, auditiva, cinestésica, olfatória, e assim por diante, que a pessoa relata ter experienciado durante uma EQM, e que posteriormente é confirmada por testemunhas independentes. Tais dados verídicos, que sugerem que os sentidos podem obter informações a distância durante uma EQM, são mais uma evidência de que a mente ampliada deve ser incluída em qualquer modelo de consciência.

\section{Estudos prospectivos de parada cardíaca}

Percepções fora do corpo que se alegam ocorrer no princípio da parada cardíaca são particularmente importantes para o nosso entendimento da consciência, porque elas abrem a possibilidade de confirmação de elas serem de fato verídicas.

Dez por cento dos pacientes com parada cardíaca têm EQM15-17,24. Desses, 30\% relatam ter uma EFC enquanto estão inconscientes e assistindo sua própria ressuscitação. Há atualmente muitos relatos retrospectivos de tais casos (para uma revisão abrangente, ver Holden $^{22}$ ) e um ou dois prováveis casos prospectivos de percepção verídica durante uma parada cardíaca. $\mathrm{O}$ caso prospectivo mais amplamente discutido, apesar de frequentemente contestado, é o de Pamela Reynolds ${ }^{25}$. Sartori ${ }^{9}$ descreve vários casos de EFC durante a inconsciência, alguns com percepção verídica, que sustentam os estudos retrospectivos anteriores. Um caso particularmente interessante do estudo AWARE é descrito em detalhe no final desta seção ${ }^{26}$.

Qualquer conclusão firme sobre a natureza verídica dessas experiências depende de se saber com precisão o momento em que ocorre. Em uma parada cardíaca, os três maiores sinais clínicos de morte clínica estão presentes: ausência de débito cardíaco, de respiração e de reflexos de tronco cerebral. O cérebro não está funcional, portanto tanto a percepção como a memória dessa percepção devem ser impossíveis. Frequentemente se argumenta que devam existir pequenas áreas de funcionamento cerebral que possa construir a experiência. No entanto, as características da experiência são tão amplas, contendo emoções, sensações visuais e auditivas e algumas vezes sensações olfatórias, que nenhuma pequena área cerebral isolada poderia gerar isso. Além disso, a memória é sempre afetada por uma disfunção cerebral grave.

A inconsciência ocorre rápido demais para que a experiência ocorra até esse momento - 11 segundos após uma parada cardíaca, sobrevém o silêncio elétrico e o cérebro está totalmente disfuncional ${ }^{27}$. As experiências são lúcidas e, portanto, não podem ocorrer durante o período de recuperação, que é sempre confusional.

Quando a ressuscitação é iniciada, é incomum que a pressão arterial aumente para mais de $30 \mathrm{mmHg}$, o que é insuficiente para o restabelecimento da circulação cerebral. O cérebro não se torna novamente funcional até que o coração volte a bater. É difícil ver como um cérebro que está intensamente prejudicado por anóxia e hipercapneia, com transmissão elétrica distorcida, seria capaz de construir a experiência tão clara, lúcida e abrangente como na EQM.

Se a percepção verídica é de fato possível durante uma parada cardíaca, então a relação entre consciência e funcionamento cerebral deve ser reavaliada. O estudo AWARE (Awareness during REsuscitation) é um estudo prospectivo que está estabelecido para testar a natureza verídica das informações colhidas durante uma EFC numa EQM. Pranchas com informações têm sido colocadas acima das camas nas áreas de ressuscitação, nos hospitais do Reino Unido, Europa e América, com o objetivo de coletar mais de 100 desses casos e testar para ver se as pranchas foram vistas. Esse é o primeiro estudo abrangente estabelecido para examinar a natureza da consciência ${ }^{28}$.

Um caso recente do estudo AWARE foi o de um paciente diabético com pouco mais de 50 anos que desenvolveu fibrilação ventricular. Ele foi trazido ao hospital por não estar se sentindo bem, com um eletrocardiograma (ECG) anormal, sendo imediatamente levado para a unidade de cateterismo cardíaco para a colocação de um stent coronariano para restaurar a circulação sanguínea em seu coração. Antes de esse procedimento poder ser realizado ele entrou em fibrilação ventricular. Isso significa que o ventrículo cardíaco apenas vibra e que o débito cardíaco cai a zero. Ele ficou imediatamente inconsciente. A equipe cardiológica, o que não é usual, utilizou-se de um desfibrilador externo automático (DEA) do tipo encontrado em shopping-centers, destinados ao uso pelo público em geral. A equipe médica colocou eletrodos, assim o DEA poderia monitorar o estado da função cardíaca. Após analisar o padrão do ritmo cardíaco, a máquina 
reconheceu fibrilação ventricular e emitiu um som alto com a instrução: "recomendo choque". O paciente então recebeu choque e a equipe médica teve que aplicar dois ciclos do protocolo de ressuscitação, o que é demandado por esse tipo de equipamento. Eles comprimiam o peito do paciente manualmente e o ventilavam com uma máscara de oxigênio; todo o processo levou de 2 a 3 minutos. A máquina, então, reanalisou o padrão do ritmo cardíaco, encontrou o paciente ainda em fibrilação ventricular e novamente recomendou que o choque fosse aplicado. Após esse segundo choque, o coração voltou a bater.

Durante o tempo em que o paciente estava em fibrilação ventricular os registros médicos indicaram que o paciente estava profundamente inconsciente e tinha iniciado o processo de morte. Após sua recuperação, quando lhe foi perguntado se ele havia vivenciado alguma coisa durante esse período, ele relatou ter visto uma garota (posteriormente ele a descreveu como um anjo) no canto do quarto acima dele. Ela fez um sinal para ele se juntar a ela e ele se viu ao seu lado olhando o processo de ressuscitação. Ele relatou ter escutado os dois comandos da máquina e ter assistido à atividade da equipe médica durante o processo de ressuscitação, e descreveu o processo corretamente.

Esse é um caso prospectivo importante, porque houve validação médica completa do estado de inconsciência do paciente durante a fibrilação ventricular e sua total consciência do processo de ressuscitação e sua própria experiência subjetiva durante esse período ${ }^{26}$.

\section{O significado dos aspectos discrepantes}

$\mathrm{O}$ fato de que muitas culturas diferentes têm conteúdo semelhante às EQM sugere a possibilidade de um funcionamento cerebral comum que ocorre nesse momento. No entanto, um plano transcendente que pode ser acessado pela experiência poderia também sugerir que a consciência é mais do que simplesmente uma questão de função cerebral. Não se encontrou um sistema explicativo mecanicista único baseado no cérebro para a grande variedade de situações em que as EQM podem ocorrer. Isso sugere que uma estrutura de consciência mais ampla, comum a todas as condições, deva ser considerada.

Em cegos congênitos a EQM parece abrir um novo caminho sensorial que obtém informação sem um órgão sensorial.

A profunda experiência espiritual na EQM sugere que a expressão da consciência do indivíduo é alterada e uma série de novas qualidades espirituais é adicionada.

Se dados verídicos podem realmente ser adquiridos durante uma EQM, isso indica que os sentidos podem obter informações a distância. Isso deve sugerir que, durante uma EQM, a mente/consciência se estende além do cérebro.

\section{Modelos de consciência}

Não existe um mecanismo cerebral aceito por meio do qual a experiência subjetiva, ou consciência, possa surgir de estruturas objetivas do cérebro. A neurociência tem avançado ao ponto em que as funções das principais áreas cerebrais são conhecidas. Sistemas de atividade durante o estado de alerta, repouso (estado-padrão) e durante o sono e sonhando, estão todos bem mapeados. A neuroimagem tem mostrado numerosas correlações entre funções e áreas cerebrais, mas esses achados são apenas correlações. Se sentimos raiva, a amígdala esquerda pode muito bem mostrar seu aumento de atividade, mas não sabemos como isso se traduz na emoção que sentimos. Ainda não há resposta para o "problema difícil" ("hard problem") de Chalmers"29,30, em que, mesmo com a nossa profunda compreensão objetiva dos mecanismos cerebrais, ainda é impossível com base neles explicar a experiência subjetiva. Modelos baseados apenas nas características físicas do cérebro, que limitam a consciência ao cérebro, estão fadados ao fracasso. Novos modelos de consciência precisam incluir a mente estendida para além do cérebro.

\section{Visão sumária dos modelos de consciência}

Os modelos de consciência têm se tornado altamente sofisticados. (Para uma revisão, ver The Handbook of Consciousness ${ }^{31}$.) Um excelente compêndio recentemente publicado no Journal of Consciousness Studies, intitulado The Singularity, tratou da questão do que acontece quando máquinas se tornam mais inteligentes que humanos e são capazes de baixar (download) a consciência em uma estrutura mecânica, com muitas referências ao The Matrix. Ele dá aos autores a oportunidade de olhar mais de perto a relação entre os sistemas neuronais do cérebro, os sistemas físicos da biologia, os sistemas evolutivos de desenvolvimento biológico, social e cultural e, finalmente, a interligação dessas ideias com a consciência universal e a forma que esta pode se ligar a esses processos físicos.

No entanto, com nosso conhecimento atual, existem três modelos básicos. Em primeiro lugar, os modelos reducionistas, que argumentam que apenas explicações materiais são válidas, excluindo a mente e a consciência como agentes causais. O modo usual de contornar essa situação é usar o conceito de "emergência", que afirma que é impossível saber quais novas propriedades irão surgir a partir de uma combinação de substâncias materiais; e que a consciência em si pode emergir com a configuração certa de elementos materiais, como a combinação de hidrogênio e oxigênio produz uma substância inteiramente nova, a água. Esse é claramente um argumento falho, pois tanto o oxigênio como o hidrogênio, quando resfriados ou comprimidos suficientemente, viram líquido. Assim, a liquidez está na sua natureza.

Outros têm discutido que essa emergência é verdadeiramente de novo, e não há maneira de prever isso. Se é assim, então o conceito de emergência não tem valor científico, ou seja, não é possível prever o que surgirá e, portanto, encontrar uma cadeia causal de explicação baseada apenas na matéria. Dehaene e Naccache ${ }^{32}$, entretanto, argumentaram que a neurociência cognitiva da consciência começa com a intenção de determinar se há uma forma sistemática de processamento de informação e uma classe específica e reprodutível de padrões de ativação neuronal que, sistematicamente (e de modo muito importante), são capazes de distinguir estados mentais de sujeitos classificados como conscientes de outros estados que não os são. Molyneux ${ }^{33}$ argumenta que isso é puramente um problema de referência e insolúvel, já que nos falta um procedimento para definitivamente descartar os candidatos explicativos neurológicos e teóricos. Ele argumenta que os correlatos neurais não deveriam ser nossa única meta, mas deveríamos vir a entender como certos tipos de mecanismos devem produzir consciência. Ele acha que isso preencherá a lacuna explicativa (explanatory gap), mas não estou certo de que isso seja um real avanço neste debate.

O próximo grupo é o de modelos que postulam que a consciência é a estrutura básica do universo e que apenas modelos que postulam a consciência como primária podem explicar com sucesso os problemas enfrentados pela neurociência atual. Tal como o primeiro modelo, esse tem uma falha inerente - o problema de uma lacuna explicativa (explanatory gap). Em ambos os casos, é necessário um pequeno milagre, seja para a consciência surgir de um processo físico, seja para a matéria surgir da consciência. Chalmers ${ }^{30}$, em um artigo que introduz o The singularity, pergunta se a inteligência artificial (IA) ou superinteligência artificial (IA+) tem o potencial de, em algum momento, chegar ao ponto em que a informação cerebral pode ser descarregada (download) em uma nova estrutura física, a qual vai absorver a nossa consciência, de modo que, com a morte do cérebro, podemos permanecer "vivos". Ele argumenta que a estrutura física que executa todos os comportamentos de uma pessoa consciente não é necessariamente consciente e que existe uma diferença entre teorias biológicas e funcionais.

O terceiro grupo, os modelos dualistas, postula que tanto a mente (consciência) quanto a matéria existem como entidades independentes, mas estão intimamente ligadas. A complexidade dos modelos está essencialmente relacionada com o grau de ligações que são permitidas. Eles podem ser absolutamente ligados de modo que cada elemento, até as partículas materiais fundamentais, tem consciência inerente a ela. Assim, de um ponto de vista, a partícula é material e de outro é um elemento da mente. Esses modelos são atraentes na medida em que superam a dificuldade da emergência da mente de uma entidade física, ou vice-versa. Mas ainda existe a dificuldade 
de explicar como a mente e o cérebro interagem e a natureza da interface mente-cérebro. Modelos dualistas ecoam na moda atual de estudar psicologia oriental e ocidental, oriental, com a meditação e o estudo de mudanças qualitativas no estado mental, e ocidental, que baseia a sua compreensão no comportamento e no conhecimento dos processos fisiológicos relacionados com o funcionamento do cérebro e, mais recentemente, o reconhecimento de que os fatores sociobiológicos são importantes.

Lockley $^{34}$ tem levado essas ideias adiante, baseado na exploração do trabalho clássico sobre a consciência de Gebser e nas ideias goethianas relacionadas com o desenvolvimento de sistemas biológicos, como elas estão incorporadas em um campo primordial da consciência. Ele sugere que essas teorias, desenvolvidas em estruturas em evolução, são responsáveis por um conjunto de influências muito mais amplas que condicionam a forma como eles evoluem e os padrões que eles tomam. Ele defende uma energia prânica cósmica que condiciona as formas evolutivas e está intimamente ligada à própria consciência universal e, dessa forma, liga consciência e matéria em um sistema único ${ }^{30}$.

\section{Teorias de campo}

Mais de $30 \%$ da população que tem experimentado uma realidade transcendente além do mundo físico descrevem-na como mais "real" que o universo diário, que é percebido como vivo e consciente. William James ${ }^{35}$, no The Varieties of Religious Experience, foi um dos primeiros a sugerir que a consciência poderia ser considerada um campo, enquanto o cérebro atua ordinariamente como um agente redutor, de modo que nossas percepções cognitivas são limitadas. Nas experiências transcendentes uma mudança no "mecanismo de filtragem" do cérebro permite que o campo mental seja estendido para o transcendente.

O maior benefício dessas teorias é que elas são não locais. A mente transcendente é universal e a mente individual pode acessá-la. Muitos dos aspectos discrepantes das EQM se enquadram facilmente nesse modelo. Considerando que é dada prioridade à transcendência no sentido causal, então cura e transformação da personalidade com dons suprassensoriais após uma EQM também podem ser explicadas. Visão mental e experiências verídicas são também mais facilmente explicadas usando um modelo holístico transcendente. E, importantemente, uma teoria de campo transcendente postula um universo que é mais próximo àquele vivenciado pelos que tiveram $\mathrm{EQM}$ do que o universo engendrado pela miscelânea de nossas limitadas teorias atuais de correlatos neurais.

Aguardamos que estudos com financiamento adequado proporcionem a tecnologia necessária [análise de eletroencefalograma (EEG) e análise do consumo de oxigênio cerebral] durante as experiências fora do corpo que ocorrem nas EQM, para confirmar se elas realmente ocorrem durante a inconsciência e, portanto, que mente e cérebro estão separados nesse momento.

\section{Referências}

1. Moody R. Life after life. Atlanta, Georgia: Mockingbird; 1975.

2. Noyes R, Slymen D. The subjective response to life-threatening danger. Omega. 1979;9:313-21.

3. Ring K. Life at death. A scientific investigation of the near death experience. New York: Coward, McCann and Georghegan; 1980.

4. Greyson B. The Near Death Experience Scale: construction, reliability, and validity. J Nerv Ment Dis. 1983;171:369-75.

5. Kellehear A. Census of non-Western near death experiences to 2005: observations and critical reflections. In: Holden JM, Greyson B, James $D$, editors. The handbook of near death experiences: thirty years of investigation. Santa Barbara, CA: Praeger; 2009. p. 135.

6. Atwater P. Coming back to life: the after-effects of the near-death experience. New York: Valentine; 1988.

7. Alexander E. Proof of heaven. London: Piatcus; 2012.
8. Fox M. Religion, spirituality and the near death experience. London and New York: Routledge; 2003.

9. Sartori P. Near death experiences of hospitalised intensive care patients. A five year clinical study. Lampeter: Edwin Mellon Press.; 2008. p. 238.

10. Fenwick P, Fenwick E. The Truth in the light: an investigation of over 300 near death experiences. London: HodderHeadline; 1995.

11. Greyson B, Kelly EW, Kelly EF. Explanatory models for the NDE experience. In: Holden JM, Greyson B, James D, editors. The handbook of near death experiences: thirty years of investigation. Santa Barbara, CA: Praeger; 2009. Chapter 10.

12. Berger RJ, Olley P, Owald I. The EEG, eye movement and dreams of the blind. Quart J Exp Psychol. 1962;14:183-6.

13. Ring K, Cooper S. Mindsight: near death and out of body experiences in the blind. Palo Alto CA: William James Center for Consciousness Studies, Institute of Transpersonal Psychology; 1999.

14. Dunne BJ, Jahn RG. Information and uncertainty in remote perception research. J Scientific Exploration. 2003;17( 2):207-41.

15. Greyson B. Near-death experiences in a psychiatric outpatient clinic population. Psychiatr Serv. 2003;54(12):1649-51.

16. Van Lommel P, van Wees R, Myers V, Elfferich I. Near death experiences in survivors of cardiac arrest: a prospective study in the Netherlands. Lancet. 2001;358:2039-45.

17. Schwaninger J, Eisenberg P, Schechtman K, Weiss A. A prospective analysis of near death experiences in cardiac arrest patients. J Near Death Studies. 2002;20(4):215-32.

18. Noyes R, Fenwick P, Holden J, Christian S. After-effects of pleasurable western adult near-death experiences. In: Holden JM, Greyson B, James $D$, editors. The handbook of near death experiences: thirty years of investigation. Santa Barbara, CA: Praeger; 2009. Chapter 3. p. 51.

19. Greyson B, Stevenson I. The phenomenology of near-death experiences. Am J Psychiatry. 1980;137(10):1193-6.

20. Tiberi E. Extra-somatic emotions. J Near-Death Studies. 1993;149-70.

21. Greyson B. Near death experiences. In: Cardena E, Lynn S, Krippner $S$, editors. Varieties of anomalous experience: examining the scientific evidence. Washington, D.C.: American Psychological Association; 2000. p. 315-52.

22. Holden J. Veridical perception in near death experience. In Holden J, Greyson B, James D, editors. The handbook of near-death experiences. Santa Barbara, CA: Praeger Publishers; 2009. Chapter 9, p. 185.

23. O’Regan B, Hirschberg C. Spontaneous remission: an annotated bibliography. Petaluma, CA: Institute of Noetic Science; 1993.

24. Parnia S, Waller DG, Yeates R, Fenwick P. A qualitative and quantitative study of the incidence, features and aetiology of near death experiences in cardiac arrest survivors. Resuscitation. 2001;48:149-56.

25. Sabom MB. The near-death experience. J Am Med Assoc. 1980;244(1):2930.

26. Parnia S. The Lazarus effect: the science that is rewriting the boundaries between life and death. London: Rider; 2013.

27. Parnia S, Fenwick P. Near death experiences in cardiac arrest: visions of a dying brain or visions of a new science of consciousness. Resuscitation. 2002;52:5-11.

28. Parnia S. Do reports of consciousness during cardiac arrest hold the key to discovering the nature of consciousness? Med Hypotheses. 2007;69(4):933-7.

29. Chalmers DJ. Facing up to the problem of consciousness. J Conscious Stud. 1995;2:200-19.

30. Chalmers DJ. The singularity of philosophical analysis. J Conscious Stud. 2010;17(9-10):7-65.

31. Velmans M, Schneider S. The blackwell companion to consciousness. Oxford: Wiley-Blackwell; 2006.

32. Dehaene S, Naccache L. Towards a cognitive neuroscience of consciousness. Basic evidence and a workspace framework. Cognition. 2001;79:1-37.

33. Molyneux B. Why the neural correlates of consciousness cannot be found. J Consciousn Stud. 2010;17(9-10):168-88.

34. Lockley MG. The evolutionary dynamics of consciousness. An integration of Estern and Western holistic paradigms. J Conscious Studi. 2010;17(9-10):66-116.

35. James W. The varieties of religious experience: a study in human nature. London \& Bombay: Longmans, Green \& Co; 1902. 